\title{
Twenty Novel Mutations in the $\alpha$-Galactosidase A Gene Causing Fabry Disease
}

\author{
Ali K. Topaloglu, ${ }^{1,2}$ Grace A. Ashley, Bingzheng Tong, ${ }^{1}$ \\ Junaid Shabbeer, ${ }^{1}$ Kenneth H. Astrin, ${ }^{1}$ Christine M. Eng, ${ }^{1,2}$ \\ and Robert J. Desnick ${ }^{1,2}$ \\ Departments of ${ }^{1}$ Human Genetics and ${ }^{2}$ Pediatrics, Mount Sinai School \\ of Medicine, New York, New York, U.S.A.
}

Accepted October 27, 1999.

\begin{abstract}
Background: Fabry disease, an X-linked inborn error of glycosphingolipid catabolism, results from the deficient activity of the lysosomal exoglycohydrolase $\alpha$-galactosidase A (EC 3.2.1.22; $\alpha$-Gal A). The nature of the molecular lesions in the $\alpha$-Gal A gene in 30 unrelated families was determined to provide precise heterozygote detection, prenatal diagnosis, and define genotype-phenotype correlations.

Materials and Methods: Genomic DNA was isolated from affected males and/or carrier females from 30 unrelated families with Fabry disease. The entire $\alpha$-Gal A coding region and flanking intronic sequences were analyzed by PCR amplification and automated sequencing. Results: Twenty new mutations were identified, each in a single family: C142R, G183D, S235C, W236L, D244H,
\end{abstract}

P259L, M267I, I289F, Q321E, C378Y, C52X, W277X, IVS4 $^{+4}$, IVS6 $^{+2}$, IVS6 $^{-1}$, 35dell3, 256dell, 892insl, $1176 \mathrm{del} 4$, and $1188 \mathrm{dell}$. In the remaining 10 unrelated Fabry families, 9 previously reported mutations were detected: M42V, R112C, S148R, D165V, N215S (in 2 families), Q99X, C142X, R227X, and 1072del3. Haplotype analysis using markers closely flanking the $\alpha-\mathrm{Gal} A$ gene indicated that the two patients with the N215S lesion were unrelated. The IVS4 ${ }^{+4}$ mutation was a rare intronic splice site mutation that causes Fabry disease. Conclusions: These studies further define the heterogeneity of mutations in the $\alpha$-Gal A gene causing Fabry disease, permit precise heterozygote detection and prenatal diagnosis, and help delineate phenotype-genotype correlations in this disease.

\section{Introduction}

Fabry disease is an X-linked inborn error of gylcosphingolipid catabolism resulting from the deficient activity of the lysosomal exoglycohydrolase, $\alpha$-galactosidase A (EC 3.2.1.22; $\alpha$-Gal A) (1). Affected individuals accumulate neutral glycosphingolipids with terminal $\alpha$-linked galactosyl moieties particularly in the plasma and in lysosomes of blood vessels, heart, liver, and kidney. In classically affected males who have markedly deficient $\alpha$-Gal A activity, the onset of the disease manifestations occurs

Address correspondence and reprint requests to: $\mathrm{Dr}$. Robert J. Desnick, Department of Human Genetics, Box 1498, Mount Sinai School of Medicine, Fifth Avenue at 100th Street, New York, NY 10029, U.S.A. Phone: 212-659-6700; Fax: 212-360-1809; E-mail: rjdesnick@msvax.mssm.edu in childhood or adolescence and is characterized by severe acroparesthesias, angiokeratoma, corneal opacities, and hypohydrosis. With advancing age, vascular disease of the heart, kidneys, and brain lead to early demise in adulthood. Asymptomatic or clinically milder hemizygotes with residual $\alpha$-Gal A activity have an attenuated form of the disease limited to cardiac involvement (2). The disease has a panethnic distribution with an estimated frequency of 1 in $\sim 40,000$ males. The full-length $\alpha$-Gal A cDNA encodes a polypeptide of 429 residues, including a 31-amino acid signal peptide (3). The $\sim 12 \mathrm{~kb} \alpha$-Gal A gene, located at Xq22.1 (Genome Database; www.gdb.org), contains seven exons, and the entire gene has been sequenced (4).

A variety of mutations in the $\alpha$-Gal A gene 


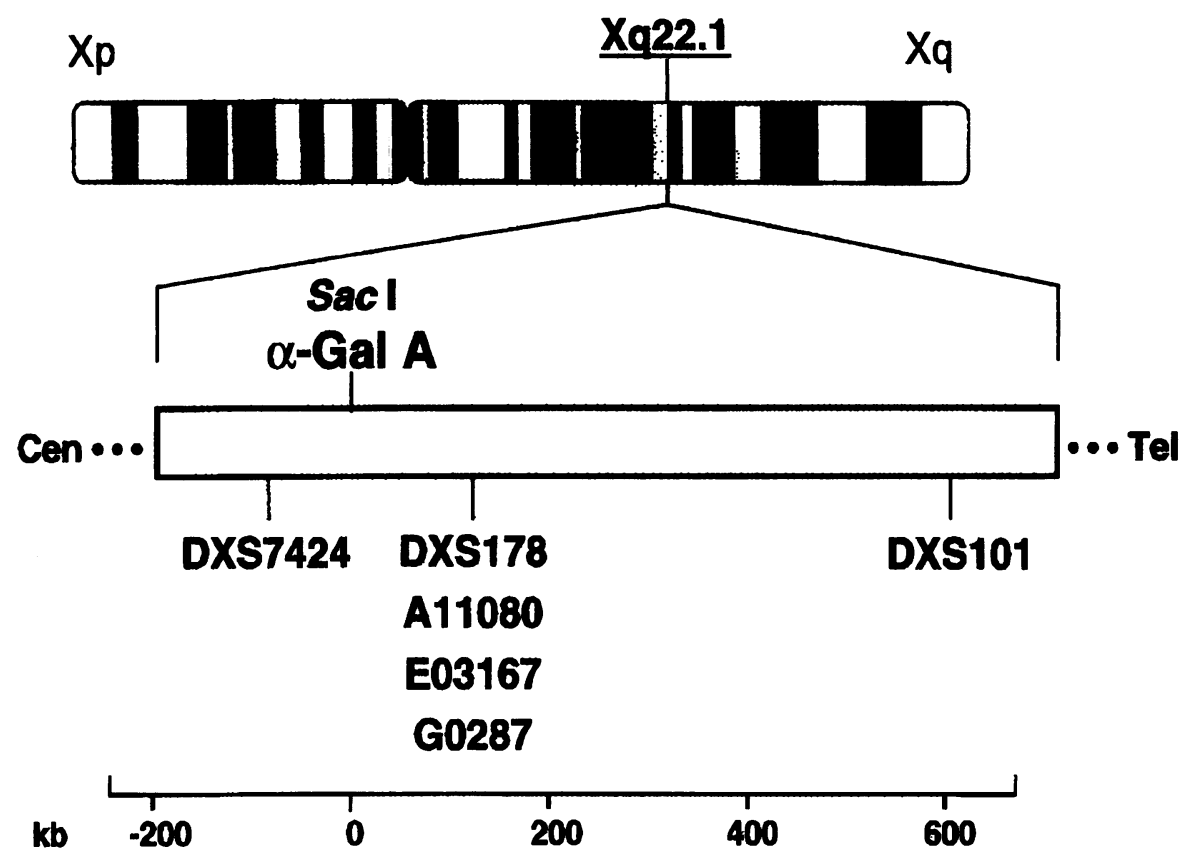

Fig. 1. Idiogram of the human G-banded X-chromosome illustrating the position of band Xq22.1 and a map of the genomic region flanking $\alpha$-Gal $A$ indicating the relative positions of the markers used for haplotype analysis.

causing Fabry disease have been described, including partial gene rearrangements, small deletions and insertions, splice-junction consensus site alterations, and various coding-region singlebase substitutions (5-7). Most mutations resulted in the classic disease phenotype; however, a few missense mutations have been detected in hemizygotes with the mild cardiac variant phenotype $(2,5,8)$. To date, most mutations have been confined to a single family with the exception of mutations involving $\mathrm{CpG}$ dinucleotides (e.g., R227Q, R227X, R342Q, and R342X) (5) and N215S, which was described in three unrelated cardiac variants (9).

To identify genotype-phenotype correlations and to permit precise heterozygote detection, the nature and frequency of the $\alpha$-Gal A mutations causing Fabry disease were determined in 30 unrelated families. In this communication, 20 new mutations are described, including a novel splice site mutation, IVS4 ${ }^{+4}$, and five missense mutations located in exon 5.

\section{Materials and Methods}

The probands from 30 unrelated families with Fabry disease, including 26 affected hemizygotes and four obligate heterozygotes, were studied with informed consent. The enzymatic diagnosis was established by determination of the 4-methylumbelliferyl- $\alpha$-Gal A activity in plasma or lympho- cytes as described previously (10). The probands were examined by the authors (C.M.E. and/or R.J.D.) or the phenotypic assessment was made by communication with the referring physicians.

For mutation analysis, genomic DNA was isolated from whole blood by standard techniques (11). The entire coding region, including all exons and flanking intronic regions, was amplified by polymerase chain reaction (PCR) from genomic DNA in four fragments as previously described (9) and each amplified genomic region was sequenced using an ABI Prism 377 DNA Sequencer (Perkin-Elmer-Cetus, Norwalk, CT). Detected mutations were resequenced in genomic DNA from the proband and/or other affected family members.

To determine if the probands with mutation N215S were related, an intragenic marker, Sac I, and closely linked markers to the $\alpha$-Gal A gene were examined. The markers were proximal flanking marker DXS7424 and the distal flanking markers DXS178, Al1080, E03167, G0287, and DXS101 (12,13). These markers span a 4-cM region between Xq22.1 and Xq22.3 (Fig. 1). All amplification reactions were carried out as previously described $(12,13)$.

\section{Results}

The $\alpha$-Gal A mutations causing Fabry disease in 30 unrelated probands were determined by mul- 
tiplex PCR amplification and automated sequencing of the entire coding sequence and adjacent intronic regions. No large $\alpha$-Gal A rearrangements (>50 bp) were observed in any of the probands following electrophoresis of the PCR products. Sequencing revealed 20 novel mutations, each in an unrelated family, including 12 coding-region missense or nonsense mutations, 3 splice site defects, 1 single-base insertion, and 4 small deletions. In addition, 9 previously published mutations were identified in 10 families, including 5 missense (M42V, R112C, S148R, D165V, and N215S), 3 nonsense (Q99X, C142X, and R227X), and 1 small deletion (1072del3). In each proband, sequencing the entire coding and adjacent flanking intronic regions revealed only a single lesion in each mutant allele. Table 1 summarizes the 20 novel and 9 recurrent mutations, the plasma $\alpha$-Gal A activity, and the phenotype of the unrelated patients.

Of the 12 novel $\alpha$-Gal A coding-region point mutations, all but one (P259L) were detected in classically affected probands, including 10 missense and 2 nonsense mutations. The missense mutations included the following: (1) a T-to-C transition of genomic nt g7323 in codon 142, resulting in a replacement of cysteine with arginine (C142R); (2) a G-to-A transition of genomic nt 8321 in codon 183, resulting in a substitution of aspartic acid for glycine (G183D); (3) a C-to-G transversion of genomic nt g10195 in codon 235, resulting in a serine-to-cysteine change (S235C); (4) a G-to-T transversion of genomic nt g10198 in codon 236, predicting a leucine-for-tryptophan substitution (W236L); (5) a G-to-C transversion of genomic nt g10221 in codon 244, resulting in a replacement of aspartic acid with histidine (D244H); (6) a C-to-T transition of genomic nt g10267 in codon 259, predicting a proline-to-leucine change (P259L); (7) a G-to-A transition of genomic nt g10292 in codon 267, resulting in a substitution of methionine with isoleucine (M267I); (8) an A-to-T transversion of genomic nt 10573 in codon 289 , resulting in an isoleucine-to-phenylalanine change (I289F); (9) a C-to-G transversion of genomic nt g10669 in codon 321, predicting a replacement of glutamine with glutamic acid (Q321E); and (10) a G-to-A transition of genomic nt gl1111 in codon 378 , resulting in a substitution of cysteine with tyrosine $(\mathrm{C} 378 \mathrm{Y})$. In addition, two novel nonsense mutations were detected, including a Cto-A transversion of genomic nt gl 335 in codon 52 predicting a cysteine to termination codon (C52X) and a G-to-A transition of genomic nt g10538 in codon 277, resulting in a tryptophan to termination codon (W277X).

Haplotype analysis of genomic DNAs from the two cardiac variant patients with the N215S mutation was performed with intragenic and flanking polymorphic markers $(12,13)$. Although the patients' DNAs were uninformative for the Sac I intragenic marker (12), their haplotypes differed at three markers, including two closely flanking markers (Fig. 1 and Table 2).

Three novel splice site mutations were detected in three classically affected males. These included a single-base alteration at the $5^{\prime}$ donor consensus splice site of intron 4 (designated IVS4 $^{+4} ; \mathrm{c} 639+4 \mathrm{~A} \rightarrow \mathrm{T}$ ), a single-base alteration at the $5^{\prime}$ donor consensus splice site of intron 6 (designated IVS6 ${ }^{+2} ;$ c999 $+2 \mathrm{~T} \rightarrow \mathrm{C}$ ), and a single-base alteration at the $3^{\prime}$ acceptor consensus site of intron 6 (designated IVS6 ${ }^{-1}$; c1000 $\mathrm{IG} \rightarrow \mathrm{A})$. Each of these lesions is predicted to cause aberrant mRNA processing and exon skipping.

Five novel small gene rearrangements causing frameshift mutations were identified, each in a classically affected proband. These include the following: (1) an insertion of a T (892insT) after genomic nt g10600 that created a TGA termination signal in codon 299 and immediate termination of the polypeptide chain; (2) a 13-base deletion (35del13) of genomic nt g1214-1249, which predicted a frameshift at codon 12 and incorporation of 105 different amino acids from residue 11 and then premature termination after residue 116; (3) a single-base deletion (256delT) of genomic nt 95155 that caused a frameshift in codon 86 and predicted the incorporation of 34 different amino acids and resulted in premature termination after residue $120 ;(4)$ a four-base deletion (1176delGAAG) of genomic nt g1115411158 that resulted in a frameshift in codon 392 , the incorporation of one different amino acid (Thr) and premature termination after codon 394; and (5) a single-base deletion (1188delC) of genomic nt gl1166 that caused a frameshift in codon 396 with the incorporation of an abnormal sequence of seven amino acids (residues 396-403) and premature termination after residue 403.

\section{Discussion}

Analysis of the $\alpha$-Gal A gene in 30 unrelated probands with Fabry disease identified 20 new mutations and 9 previously reported lesions. Se- 
Table 1. Mutations and clinical features of $\mathbf{3 0}$ unrelated Fabry disease hemizygotes or heterozygotes

\begin{tabular}{|c|c|c|c|c|c|}
\hline Patient & Location & $\begin{array}{l}\text { Allele codon or } \\
\text { cDNA nucleotide }{ }^{a}\end{array}$ & $\begin{array}{l}\text { Genomic nucleotide } \\
\text { change }^{b}\end{array}$ & $\begin{array}{l}\text { Plasma } \alpha-G a l ~ A \\
\text { activity (U/ml) }\end{array}$ & $\begin{array}{c}\text { Clinical } \\
\text { phenotype }^{c}\end{array}$ \\
\hline \multicolumn{6}{|c|}{ Novel mutations } \\
\hline \multicolumn{6}{|c|}{ Missense } \\
\hline 1 & Ex 3 & $\mathrm{Cl} 142 \mathrm{R}$ & g7323T $\rightarrow C$ & 1.6 & Classic \\
\hline 2 & Ex 4 & G183D & g8321G $\rightarrow A$ & 1.0 & Classic \\
\hline 3 & Ex 5 & $\mathrm{~S} 235 \mathrm{C}$ & g10195C $\rightarrow G$ & 1.3 & Classic \\
\hline 4 & $\operatorname{Ex} 5$ & W236L & $\mathrm{gl} 10198 \mathrm{G} \rightarrow \mathrm{T}$ & 0.8 & Classic \\
\hline 5 & Ex 5 & D244H & $\mathrm{g} 10221 \mathrm{G} \rightarrow \mathrm{C}$ & - & Het/classic \\
\hline 6 & Ex 5 & P259L & $\mathrm{g} 10267 \mathrm{C} \rightarrow \mathrm{T}$ & 0.3 & Cardiac \\
\hline 7 & Ex 5 & M267I & $\mathrm{gl} 10292 \mathrm{G} \rightarrow \mathrm{A}$ & 0.2 & Classic \\
\hline 8 & Ex 6 & $\mathrm{I} 289 \mathrm{~F}$ & $\mathrm{~g} 10573 \mathrm{~A} \rightarrow \mathrm{T}$ & 1.9 & Classic \\
\hline 9 & Ex 6 & Q321E & $\mathrm{gl} 10669 \mathrm{C} \rightarrow \mathrm{G}$ & 0.3 & Classic \\
\hline 10 & Ex 7 & C378Y & $\mathrm{g} 11111 \mathrm{G} \rightarrow \mathrm{A}$ & - & Het/classic \\
\hline \multicolumn{6}{|c|}{ Nonsense } \\
\hline 11 & Ex 1 & $\mathrm{C} 52 \mathrm{X}$ & g1335C $\rightarrow A$ & 13.3 & Het/classic \\
\hline 12 & Ex 1 & W277X & $\mathrm{gl0538G} \rightarrow \mathrm{A}$ & 0.9 & Classic \\
\hline \multicolumn{6}{|c|}{ Splice Site } \\
\hline 13 & Int 4 & $\mathrm{IVS}^{+4}$ & $\mathrm{~g} 8416 \mathrm{~A} \rightarrow \mathrm{T}$ & 1.0 & Classic \\
\hline 14 & Int 6 & IVS6 $^{+2}$ & $\mathrm{~g} 10709 \mathrm{~T} \rightarrow \mathrm{C}$ & 0.6 & Classic \\
\hline 15 & Int 6 & IVS6 $^{-1}$ & $\mathrm{~g} 10977 \mathrm{~T} \rightarrow \mathrm{C}$ & - & Classic \\
\hline \multicolumn{6}{|c|}{ Rearrangements } \\
\hline 16 & Ex 1 & 35 dell 3 & 1214delGCGCGCTTGCGCT & 0.5 & Classic \\
\hline 17 & Ex 2 & 256dell & 5151 delT & 1.0 & Classic \\
\hline 18 & Ex 6 & 892ins l & 10600insT & 1.3 & Classic \\
\hline 19 & Ex 7 & 1176del4 & 11154 delGAAG & 0.4 & Classic \\
\hline 20 & Ex 7 & 1188dell & $11166 \mathrm{delC}$ & 0.2 & Classic \\
\hline \multicolumn{6}{|c|}{ Previously reported mutations } \\
\hline \multicolumn{6}{|c|}{ Missense } \\
\hline 21 & Ex 1 & M42V & $\mathrm{gl} 303 \mathrm{~A} \rightarrow \mathrm{C}$ & 6.8 & Het/classic \\
\hline 22 & Ex 2 & R112C & g5233C $\rightarrow T$ & 0.2 & Classic \\
\hline 23 & Ex 3 & S148R & g7343T $\rightarrow G$ & 0.4 & Classic \\
\hline 24 & Ex 3 & Dl65V & $\mathrm{g} 7393 \mathrm{~A} \rightarrow \mathrm{T}$ & - & Classic \\
\hline 25 & $\operatorname{Ex} 5$ & N215S & g10135A $\rightarrow G$ & 0.5 & Cardiac \\
\hline 26 & Ex 5 & N215S & g10135A $\rightarrow G$ & 6.2 & Het/cardiac \\
\hline \multicolumn{6}{|c|}{ Nonsense } \\
\hline 27 & Ex 2 & Q99X & $\mathrm{g} 5194 \mathrm{C} \rightarrow \mathrm{T}$ & 0.5 & Classic \\
\hline 28 & $\operatorname{Ex} 3$ & C142X & g7325C $\rightarrow A$ & 0.2 & Classic \\
\hline 29 & Ex 5 & R227X & $\mathrm{g} 10170 \mathrm{C} \rightarrow \mathrm{T}$ & 0.1 & Classic \\
\hline \multicolumn{6}{|c|}{ Rearrangements } \\
\hline 30 & Ex 7 & 1072 del 3 & 11050delGAG & 0.2 & Classic \\
\hline
\end{tabular}

Het, heterozygote.

${ }^{a}$ cDNA reference sequence (10).

${ }^{b}$ Genomic reference sequence (2).

${ }^{c}$ Classic or cardiac phenotype. 
Table 2. Haplotype analysis of Fabry disease patients with mutation $\mathrm{N} 215 \mathrm{~S}$

\begin{tabular}{lcc}
\hline Marker & Patient 25 & Patient 26 \\
\hline DXS7424 & $\mathbf{7}$ & $\mathbf{1}, 5$ \\
DXS8096 & 1 & 1,2 \\
DXS178 & 3 & $\mathbf{2 , 5}$ \\
A11080 & 3 & 3,3 \\
E03167 & 3 & 1,3 \\
G0287 & 2 & 2,2 \\
DXS101 & $\mathbf{1}$ & $\mathbf{4}, 5$ \\
\hline
\end{tabular}

Markers with different alleles in the two patients are shown in bold.

quencing of the entire coding region and flanking intronic sequences revealed only one alteration per mutant allele. In addition, all 28 mutations were confirmed in genomic DNA from other hemizygous or heterozygous family members (data not shown).

To date, most of the mutations in the $\sim 12$ $\mathrm{kb} \alpha$-Gal A gene causing Fabry disease have been identified in single families (i.e., familyspecific). Of the mutations detected in several families, many have occurred at $\mathrm{CpG}$ dinucleotides, known hotspots for mutations (14). Notably, none of the new missense or nonsense mutations occurred at the $14 \mathrm{CpG}$ dinucleotides of the $\alpha$-Gal A coding sequence, and each was a "private" mutation that occurred in a single family. Among the 10 families with the 9 previously reported mutations, there were 5 missense and 3 nonsense mutations. Of these, only R112C and R227X occurred at CpG dinucleotides. Haplotype analysis of the $\alpha$-Gal A flanking region in the two families with the common non-CpG N215S lesion revealed different alleles at three of the seven polymorphisms flanking the $\alpha$-Gal A gene (Fig. 1 and Table 2). The fact that two of three of these informative markers were close to the $\alpha$-Gal A gene $(<100 \mathrm{~kb})$ suggested that these patients were not related.

Three mutations $\left(\mathrm{IVS}^{+4}{ }^{+}\right.$IVS6 $^{+2}$, and IVS6 $^{-1}$ ) that altered the processing of the $\alpha$-Gal A transcript were identified in three classically affected hemizygotes. The IVS4 ${ }^{+4}$ splice site mutation is the first example of a disease-causing mutation in the fourth intronic position of the $5^{\prime}$ donor consensus splice site sequence ([C/A]AG- gt[a/g]agt) that causes the classic Fabry phenotype. Of the nine previously reported splice site mutations, all involved single-base substitutions at the canonical ag or gt dinucleotides at intronexon junctions (Human Gene Mutation Database; www.uwcm.ac.uk/uwcm/mg/hgmd0.html). This mutation introduces a thymidine in intronic position 4, which is a rare event and which predicts the possible skipping of exon 4 and a frameshift mutation, resulting in an aberrant and truncated polypeptide. The two IVS6 lesions involved a T-to- $C$ transition in position 2 of the $5^{\prime}$ donor consensus sequence (IVS6 ${ }^{+2}$ ) and a G-to-C transversion in the last nt of intron 6 at the $3^{\prime}$ acceptor consensus splice site (IVS6 ${ }^{-1}$ ). These intronic mutations in the $5^{\prime}$ donor and $3^{\prime}$ acceptor consensus sequences are known to cause aberrant splicing (8).

Of the gene rearrangements, the $35 \mathrm{del} 13$ mutation involved the deletion of a GC-rich sequence GCGCGCTTGCGCT, which contained five GC repeats. Both breakpoints were immediately flanked by the trinucleotide short repeats GCT and its inverse TCG, which suggested that illegitimate recombination or a slipped mispairing event may have been the deletional mechanism. In addition, two small deletions were detected within a 13-base pair region in exon 7 (1176del4 and 1181dell). The identification of these deletions further supports the previous suggestion that exon 7 is a region prone to small gene rearrangements (7).

All of the novel mutations were identified in classically affected probands or in obligate heterozygotes whose affected sons had the classic phenotype with the exception of P259L. This missense mutation was identified in a 38year-old cardiac variant with left heart enlargement and minimal proteinuria. In addition, the two patients with the previously identified N215S mutation also had the cardiac variant phenotype.

In summary, the identification of these 20 new mutations further documents the remarkable genetic heterogeneity of the $\alpha$-Gal A lesions causing Fabry disease. Mutation analysis is important for each Fabry family, as the detection of these mutations permits precise diagnosis of heterozygous carriers of this $\mathrm{X}$-linked recessive gene and provides the ability to perform molecular prenatal diagnoses. In addition, the detection of these mutations is useful for evaluation of genotype-phenotype correlations as well as for insights into the enzyme's structure-function relationships. 


\section{Acknowledgments}

We thank the following physicians for referral of patients and/or diagnostic specimens: Drs. G. Appel, D. Barfield, S. Cederbaum, M. C. Clarke, J. Daven, H. Goldman, G. M. Grasso, B. Kornhall, T. W. Kurczynski, M. Kushigemachi, R. Lee, A. Linder, V. Moore, J. Murphy, L. Olson, R. Pyeritz, S. Phadke, L. Shapiro, J. Sloand, and D. Suhrbier. This work was supported in part by grants from the National Institutes of Health, including a Merit Award (5 R37 DK34045), a grant (5 MO1 RR00071) for the Mount Sinai General Clinical Research Center, and a grant (5 P30 HD28822) for the Mount Sinai Child Health Research Center.

\section{References}

1. Desnick RJ, Ioannou YA, Eng CM. (1996) $\alpha$-Galactosidase A deficiency: Fabry disease. In: Scriver $\mathrm{CR}$, Beaudet AL, Sly WAS, Valle D (eds). The Metabolic and Molecular Basis of Inherited Diseases, 7th ed. McGraw-Hill, New York, pp. 2741-2784.

2. von Scheidt W, Eng CM, Fitzmaurice TF, et al. (1991) An atypical variant of Fabry's disease with manifestations confined to the myocardium. N. Engl. J. Med. 324: 395-399.

3. Bishop DF, Calhoun DH, Bernstein HS, Hantzopoulos P, Quinn M, Desnick RJ. (1986) Human $\alpha$-galactosidase A: nucleotide sequence of a cDNA clone encoding the mature enzyme. Proc. Natl. Acad. Sci. U.S.A. 83: 4859-4863.

4. Kornreich R, Desnick RJ, Bishop DF. (1989) Nucleotide sequence of the human $\alpha$-galactosidase A gene. Nucl. Acids Res. 17: 3301-3302.

5. Eng CM, Niehaus DJ, Enriquez A, Burgett TS, Ludman MD, Desnick RJ. (1994) Fabry disease: twenty-three mutations including sense and antisense CpG alterations and identification of a deletion hot-spot in the $\alpha$-galactosidase A gene. Hum. Mol. Genet. 3: 1795-1799.
6. Eng CM, Desnick RJ. (1994) Molecular basis of Fabry disease: mutations and polymorphisms in the human $\alpha$-galactosidase A gene. Hum. Mutat. 3: 103-111.

7. Eng CM, Ashley GA, Burgert TS, Enriquez AL, D'Souza M, Desnick RJ. (1997) Fabry disease: thirty-five mutations in the $\alpha$-galactosidase $A$ gene in patients with classic and variant phenotypes. Mol. Med. 3: 174-182.

8. Sakuraba H, Oshima A, Fukuhara Y, et al. (1990) Identification of point mutations in the $\alpha$-galactosidase A gene in classical and atypical hemizygotes with Fabry disease. Am. J. Hum. Genet. 47: 784789.

9. Eng CM, Resnick-Silverman LA, Niehaus DJ, Astrin KH, Desnick RJ. (1993) Nature and frequency of mutations in the $\alpha$-galactosidase A gene that cause Fabry disease. Am. J. Hum. Genet. 53: 11861197.

10. Desnick RJ, Allen KY, Desnick SJ, Raman MK, Bernlohr RW, Krivit W. (1973) Fabry disease: enzymatic diagnosis of hemizygotes and heterozygotes. $\alpha$-Galactosidase activities in plasma, serum, urine and leukocytes. J. Lab. Clin. Med. 81: 157171.

11. Sambrook J, Fritsch EF, Maniatis T. (1989) Molecular Cloning. A Laboratory Manual. Cold Spring Harbor Laboratory Press, Cold Spring Harbor, NY.

12. Kornreich R, Astrin KH, Desnick RJ. (1992) Amplification of human polymorphic sites in the $\mathrm{X}$ chromosomal region $\mathrm{q} 21.33$ to q24: DXS17, DXS87, DXS287, and $\alpha$-galactosidase A. Genomics 13: $70-74$.

13. Caggana M, Ashley GA, Desnick RJ, Eng CM. (1997) Fabry disease: molecular carrier detection and prenatal diagnosis by analysis of closely linked polymorphisms at Xq22.1. Am. J. Med. Genet. 71: 329-335.

14. Cooper C, Youssoufian H. (1988) The CpG dinucleotide and human genetic disease. Hum. Genet. 78: 151-155. 